\begin{tabular}{|c|c|}
\hline \multirow{3}{*}{ 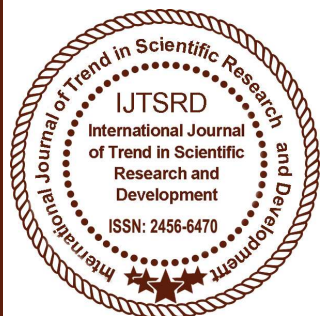 } & $\begin{array}{l}\text { International Journal of Trend in Scientific } \\
\text { Research and Development (IJTSRD) }\end{array}$ \\
\hline & International Open Access Journal \\
\hline & ISSN No: 2456 - 6470 | www.ijtsrd.com | Volume - 2 | Issue - 2 \\
\hline
\end{tabular}

\title{
A Study on Orbital Mechanics
}

\author{
N. Gayathri \\ Assistant Professor, Sri Krishna Arts and Science \\ College, Coimbatore, Tamilnadu, India
}

\author{
S. Mahalakshmi \\ Student, Sri Krishna Arts and Science College, \\ Coimbatore, Tamilnadu, India
}

\begin{abstract}
Space craft to solar system bodies must deal with multiple deviations under the in uence of gravity. The motion of satellite in one of the types of conic section strongly depends upon escape velocity. The goal of this paper is to nd the trajectory of satellite by analysing orbit's eccentricity and or-bital parameters. This paper also focuses on the derivation of general orbit equation from the base called Newton's law of motion and Newton's law of gravitation.
\end{abstract}

Keywords: Eccentricity, Flight path angle, True anomaly, Perigee and Apogee radius, Trajectory.

\section{INTRODUCTION}

Mechanics is a branch of science concerned with the relationship between force, displacements, energy and their e ects on physical bodies on their environment. The classical mechanics is one of the sub- elds of mechanics. Classical mechanics is concerned with the some physical laws describing the movement of bodies under the in uence of forces.

\section{ORBITAL MECHANICS}

Orbital mechanics is also known as astrodynamics. It is the application of celestial mechanics concerning the motion of rockets and other spacecraft. It is concerned with motion of bodies under the in uence of gravity, including both spacecraft and natural astronomical bodies such as star, planets, moons and comets.

\subsection{TRAJECTORIES:}

Three general types of paths are possible under the gravitational in uence.

An elliptical orbit is a orbit with an eccentricity of less than 1. This includes the special case called circular orbit and its eccentricity is equal to 0 .

This paper examines the classifications, cause; types and the possible solutions of conflict in organization.

This discovered that conflict generates considerable ambivalence and leave many practitioners and scholars quite uncertain about how best to cope with it. Conflicts are inevitable in human life and also inevitable in organizations even between countries. Conflict occurs in organizations as a result of competition for supremacy, scarcity of economic resource and leadership style. The study also revealed that conflict in organization could be constructive or destructive which can lead to low production or good solution to production.

A hyperbolic trajectory is the trajectory of any body around a cen-tral mass with more enough speed to escape the central object's grav-itational pull. The orbital eccentricity will be greater than one

A parabolic trajectory is an orbit whose eccentricity will be equal to 1 . A body travelling along an escape orbit will coast along a parabolic trajectory to in nity and never return. 


\subsubsection{ESCAPE VELOCITY:}

Escape velocity is the velocity required at a given position to establish a parabolic path. Velocities greater than escape velocity result in hyperbolic orbits. Lower velocities result in closed elliptical orbits.

\subsection{ORBITAL PARAMETERS:}

Apogee (q) refers to the farthest distance between satellite and the Earth.

Perigee (p) refers to the closest distance between satellite and the Earth.

\section{ORBIT EQUATION}

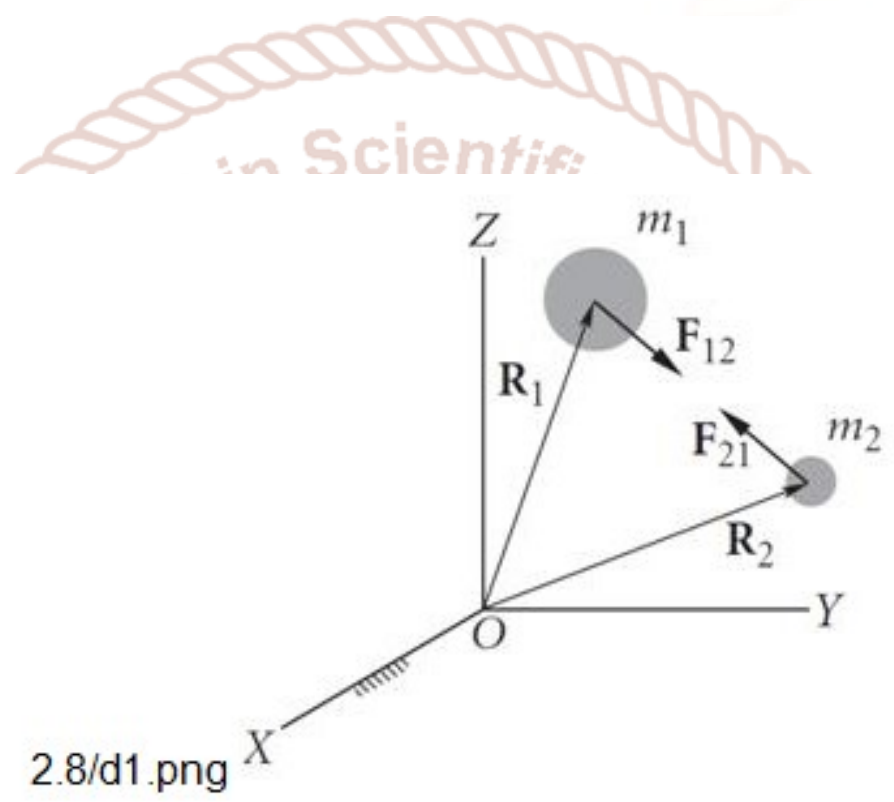

Two objects are located in inertial frame of reference XYZ. Each of the bod-ies is acted upon by the gravitational attraction of the other. $F_{12}$ is the force exerted on $m_{1}$ by $m_{2}$, and $F_{21}$ is the force exerted on $m_{2}$ by $m_{1}$. The position vector $R_{G}$ of the center of mass $G$ is de ned by,

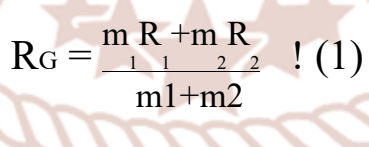

Absolute velocity, $\mathrm{v} \underset{\mathrm{G}}{=} \underset{\mathrm{G}}{\mathrm{R} 0}=\frac{\mathrm{m} 1 \mathrm{R} 10+\mathrm{m} 2 \mathrm{R} 20}{\mathrm{~m} 1+\mathrm{m} 2}$

Absolute acceleration, a

$$
\frac{=R^{00}=m_{1} R_{1} 00+m_{2} R_{2} 00}{m_{1}+m_{2}}
$$

The term absolute means that the quantities are measured relative to an inertial frame of reference.

Let $r$ be position vector of $m_{2}$ relative to $m_{1}$.

$$
\mathrm{r}=\mathrm{R}_{2} \quad \mathrm{R}_{1} !(4)
$$

Let $\mathrm{uc}_{\mathrm{r}}$ be the unit vector pointing from $\mathrm{m}_{1}$ towards $\mathrm{m}_{2}$,

$$
\mathrm{u}=\underline{\mathrm{r}} !(5)
$$




\section{$\mathrm{c}_{\mathrm{r}} \mathrm{r}$}

where $r=k r k$ is the magnitude of $r$.

The force exerted on $\mathrm{m}_{2}$ by $\mathrm{m}_{1}$ is,

$$
F_{21}=\frac{G m_{1} m_{2}\left(u_{b}\right)}{r^{2}}=\frac{G m_{1} m_{2}\left(u_{b}\right)}{r^{2}} !(6)
$$

where $\mathrm{uc}_{\mathrm{r}}$ shows that the force vector $\mathrm{F}_{21}$ is directed from $\mathrm{m}_{2}$ towards $\mathrm{m}_{1}$.

Applying Newton's second law of motion on body $\mathrm{m}_{2}$ is $\mathrm{F}_{21}=\mathrm{m}_{2} \mathrm{R}_{2}{ }^{00}$, where $\mathrm{R}_{2}{ }^{00}$ is the absolute acceleration of $\mathrm{m}_{2}$.

$$
\frac{G m_{1} m_{2}\left(y_{0}\right)=m_{2} R \times 0}{r^{2}} !(7)
$$

By Newton's third law of motion, $F_{12}=F_{21}$, so for $m_{1}$ we have,

$$
\frac{G m_{1} m_{2}\left(u_{b_{r}}\right)}{r^{2}}=m^{1} R^{00} !(8)
$$

Equations (7)and (8) are the equations of motion of two bodies in inertial space. By adding, we get $m_{1} R_{1}{ }^{00}+m_{2} R_{2}{ }^{00}=0 !(9)$.

According to Equation(3), the acceleration of the center of mass $\mathrm{G}$ of the system of two bodies $m_{1}$ and $m_{2}$ is zero.

Multiplying Equation (7) by $m_{1}$ and Equation (8) by $m_{2}$, we get

$$
\begin{aligned}
\frac{G m^{2} m_{2}\left(Y_{0}\right)}{1_{2}} & =m m^{1}{ }^{2}, ! \\
\frac{G m_{1} m}{b} & =m_{1} m_{2} R \quad 100 !
\end{aligned}
$$

Subtracting the equation(11) from (10) yields

$$
\begin{gathered}
m m_{2}\left(R_{2}^{00} R^{00}\right)=\frac{G m_{1} m_{2}}{r^{2}}\left(m_{1}+{ }_{-} m_{2}\right) u_{L_{-}} u_{-}(12) \\
\text { factor } m_{1} m_{2} \\
r^{\prime \prime}=\frac{G\left(m_{1}+m_{2}\right)}{r^{2}} u_{c} !(13)
\end{gathered}
$$

Let the gravitational parameter be de ned as

$=\mathrm{G}\left(\mathrm{m}_{1}+\mathrm{m}_{2}\right) !(14)$

Using Equation (14) and Equation (5), we can write Equation (13) as

$r^{\prime \prime}=\quad r 3 !(15)$ 
International Journal of Trend in Scientific Research and Development (IJTSRD) ISSN: 2456-6470

This is the second order di erential equation that governs the motion of $m_{2}$ relative to $m_{1}$.

Let $r_{1}$ and $r_{2}$ be the position vectors of $m_{1}$ and $m_{2}$ relative to the center of mass $G$. The equation of motion of $m_{2}$ relative to the center of mass is

$$
\frac{\mathrm{Gm}_{1} \mathrm{~m}_{2}\left(\mathrm{u}_{\mathrm{b}_{n}}\right)}{\mathrm{r}^{2}}=\mathrm{m}^{2} \mathrm{r}^{00} !(16)
$$

where $r$ is the position vector of $m_{2}$ relative to $m_{1}$. In terms of $r_{1}$ and $r_{2}$,

$\mathrm{r}=\mathrm{r}_{2} \quad \mathrm{r}_{1}$

Since the position vector of the center of mass relative to itself is zero, we get,

$$
\begin{aligned}
& m_{1} r_{1}+m_{2} r_{2}=0 \\
& r_{1}={ }_{m_{1}^{2}} r_{2} \\
& r=m_{1}^{m_{2}+m_{2}} r_{2} m_{1}
\end{aligned}
$$

Substituting this back into Equation (16) and using $\left(\mathrm{uc}_{\mathrm{r}}\right)=\mathrm{r}_{2}=\mathrm{r}_{2}$, we get

$$
\frac{\mathrm{Gm}_{3} \mathrm{~m}_{2} r=m}{\left(\mathrm{~m}_{1}+\mathrm{m}_{2}\right)^{2} \mathrm{r}_{2}{ }^{3}} 22_{2}{ }^{2}
$$

Simplifying,

let $\quad v=\left(\frac{\left.m_{1},\right)^{3}}{m_{1}+m_{2}}\right.$

$$
\left(\frac{m_{1}}{m_{1}+m_{2}}\right)_{\underset{r_{2}}{r}}^{3}=r_{2}
$$

$$
\mathrm{r}^{0} \mathrm{r}_{2}=\mathrm{r}^{00}{ }_{2}
$$

Similarly, the equation of motion of $m_{1}$ relative to the center of mass is found to be

$$
\text { where } 00=\left(\frac{m_{2}}{m_{1}+m_{2}}\right)^{3}
$$

$$
{ }_{\mathrm{r}^{20}}^{\mathrm{OO}} \mathrm{r}_{1}=
$$

The angular momentum of body $m_{2}$ relative to $m_{1}$ is the moment of $m_{2}$ 's relative linear momentum $m_{2} r^{0}$

$$
\mathrm{H}_{2=1}=r \times \mathrm{m}_{2} \mathrm{r}^{0}
$$

Divide this equation by $\mathrm{m}_{2}$ and let $\mathrm{h}=\mathrm{H}_{2=1}=\mathrm{m}_{2}$, so that

$$
\mathrm{h}=\mathrm{r} \times \mathrm{r}^{0} \text { ! }
$$

$\mathrm{h}$ is the relative angular momentum of $\mathrm{m}_{2}$ per unit mass, that is, the speci $\mathrm{c}$ relative angular momentum. Taking the time derivative of $h$ yields 
$\frac{d}{d d t} h=r^{0} \times r^{0}+r_{r} r^{\infty}$

But $r^{0} \times r^{0}=0$. According to Equation (15) $r^{\prime \prime}={ }_{r 3} r$, we have

$$
\begin{aligned}
r \times r^{00}= & r \times(r \bar{r})=r_{3}(r \bar{x} r)=0{ }_{\text {codt }}^{d} h \\
& =0 !(19)
\end{aligned}
$$

the unit vector,

$$
\mathrm{hb}=\frac{\mathrm{h}_{\mathrm{h}}}{\mathrm{m}} \mathbf{( 2 0 )}
$$

Let us divide the relative velocity vector $r^{0}$ into components $v_{r}=v_{r} u b_{r}$ and $v_{p}=v_{p} u b_{p}$, where $u b_{r}$ and $\mathrm{ub}_{\mathrm{p}}$ are radial and perpendicular unit vector

$$
h=r u b_{r} \times\left(v_{r} u b_{r}+v_{p} u b_{p}\right)=r v_{p} h^{b} h=r v_{p} !(21)
$$

The angular momentum depends only on the perpendicular component of the relative velocity.

During the di erential time interval $d t$ the position vector $r$ sweeps out an area dA.

From the gure the triangular area $\mathrm{dA}$ is given by

\section{8/d2.png}

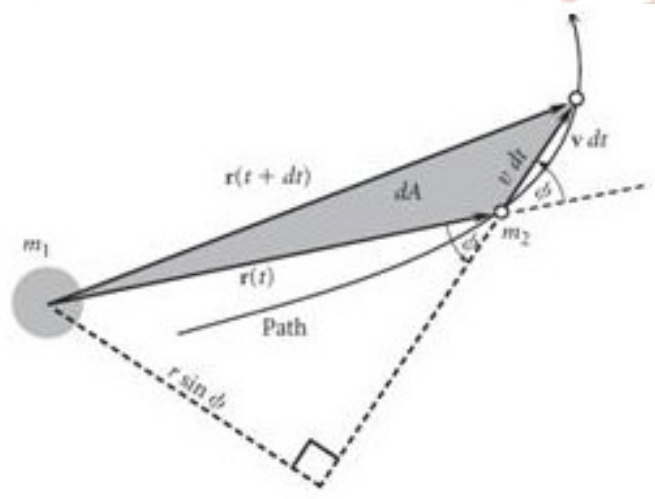

$$
\mathrm{dA}=\frac{1}{2}_{2} \times \text { base } \times \text { height }=\frac{1}{2} \times \mathrm{vdt} \times \mathrm{r} \sin =\frac{1}{2} \mathrm{r}(\mathrm{v} \sin \mathrm{dt})=1_{2} \mathrm{rvpdt}
$$

Therefore, using Equation (21) we get

$$
{ }_{\mathrm{dA}}^{\mathrm{dA}}=\frac{\mathrm{h}}{2} \quad !(22)
$$

The vector identity also known as the bac - cab rule:

$$
A A \times(B \times C)=B(A: C) C(A: B) !(23) r: r=r^{2}
$$

$$
\mathrm{dt}^{\mathrm{d}}(\mathrm{r}: \mathrm{r})=2 \mathrm{r} \frac{\mathrm{dr}}{\mathrm{dt}}
$$

But

$$
d^{d}(r: r)=r_{d t}^{d}+{ }_{d t}^{d} r=2 r \frac{d r}{d t}
$$

Thus, we obtain the identity 
International Journal of Trend in Scientific Research and Development (IJTSRD) ISSN: 2456-6470

$$
r: r^{0}=r: r^{0} \quad !(25 a)
$$

Since $r^{0}=v$ and $r=k r k$, this can be written alternatively as

$$
r: v=k r k \frac{k d r}{d t} k
$$

Let us take the cross product on both sides of Equation (15) $r "={ }_{r 3} r$ with the speci $c$ angular momentum h:

$$
r^{\prime \prime} \times h=\underline{r_{3}} r \times h
$$

Since ${ }_{\text {dt }}^{d}\left(r^{0} \times h\right)=r^{00} \times h+r^{0} \times h^{0}$, the left-hand side can be written

$$
r^{00} \times h=d t\left(r^{0} \times h\right)-r^{0} \times h^{0}
$$

But according to Equation (19), the angular momentum is constant $\left(\mathrm{h}^{0}=0\right)$, so

$$
r^{00} \times h=d t{ }^{d}\left(r^{0} \times h\right)
$$

The right-hand side of Equation (26) can be transformed into:

$$
\begin{aligned}
& r_{3}^{1} r \times h=r^{1}\left[\left(r \times\left(r \times r^{0}\right)\right)\right. \text { Scientit } \\
& =r_{3}^{1}\left[r\left(r: r^{0}\right) r^{0}(r: r)\right] \\
& =r^{1}\left[r\left(r^{0}\right) r^{0}\left(r^{2}\right)\right] \\
& \left(r^{0} \quad r^{0} r\right)=6470=r
\end{aligned}
$$

But

$$
\frac{d r}{d t(r)}=\frac{\left(r^{0} r r^{0}\right)}{r^{2}}=\left(\frac{r_{r}^{0} r^{0} r}{r^{2}}\right)
$$

Therefore

$$
\left.{ }_{\leftarrow 3}^{1} r \times h=\underset{d t}{d} \stackrel{r}{(} r\right) !(28)
$$

Substituting Equations (27) and (28) into Equation (26), we get

$$
\begin{aligned}
& d r^{d}\left(r^{0} \times h\right)=d t\left(\frac{r}{r-}\right) \\
& d\left(r^{0} \times h-\quad \underline{r}\right)=0
\end{aligned}
$$

Taking the dot product of $h$ on both sides of Equation (29) yields 


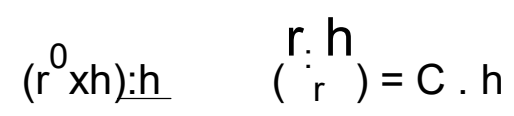

Since $r^{\prime} x h$ is perpendicular to both $r^{\prime}$ and $h$, so that $\left(r^{\prime} \times h\right) . h=0$. Likewise, since $h=r x$ $r^{\prime}$ is perpendicular to both $r$ and $r^{\prime}$, it follows that $r . h=0$. Therefore, we have $C . h=0$, i.e., $\mathrm{C}$ is perpendicular to $\mathrm{h}$, which is normal to the orbital plane.

Rearranging Equation (29)

$$
\underline{r}_{r}+e=1\left(r^{0} \times h\right) !(30)
$$

where $\mathrm{e}=\mathrm{C}=$. The vector $\mathrm{e}$ is called the eccentricity. The line de ned by the $\mathrm{e}$ is commonly called the apse line. o obtain a scalar equation, let us take the dot product of both sides of Equation (30) with $r$

$$
r \cdot r+e=1\left(r:\left(r^{0} \times h\right)\right) !(31)
$$

we know the identity

to obtain

$$
A:(B \times C)=(A \times B): C
$$

$$
r:\left(r^{0} \times h\right)=\left(r \times r^{0}\right): h=h: h=h^{2} !(32)
$$

Substituting this expression into the right-hand side of Equation (31), and substituting $r$. $r=r^{2}$ on the left yields

$$
r+r: e=h_{2} \quad(33)
$$

From the de nition of dot product we have

$$
r: e=r e \cos
$$

In terms of the eccentricity and the true anomaly, we may write Equation (33) as

$$
\begin{gathered}
r+r e \cos =h_{2} \\
r=\underline{h^{2}} \frac{1}{1+e \cos }
\end{gathered}
$$

This is the orbit equation, and it de nes the path of the body $m_{2}$ around $m_{1}$, relative to $m_{1}$.

\subsection{Flight path angle:}

The ight path angle is the angle that the velocity vector $v=r$ makes with the normal to the position vector.

$$
\tan =\underset{v_{p}}{\stackrel{v}{r}}
$$


International Journal of Trend in Scientific Research and Development (IJTSRD) ISSN: 2456-6470

$$
\tan =\frac{e \sin }{1+e \cos }
$$

\subsection{Period of orbit (ellipse):}

At periapsis, $\quad=0^{\circ}$

$$
r_{p}=\frac{h^{2}}{1+e} \frac{1}{e}(34)
$$

At apoapsis, $=180^{\circ}$

$$
r_{a}=\frac{h^{2}}{1} \frac{1}{e} !(35)
$$

Let $2 a$ be the distance between from periapsis $P$ to apoapsis $A, 2 a=r_{p}+r_{a}$

$$
\begin{gathered}
A, 2 a=r_{p}+r_{a} \\
2 a=\frac{h^{2}}{1+e} \frac{1}{1+\frac{h^{2}}{1}} \frac{1}{e} \\
a=\frac{h^{2}}{1} \frac{1}{e^{2}} \\
b=a p \\
1 e^{2}
\end{gathered}
$$

Area of ellipse $=A=a b$

By Kepler's second law,

$$
\begin{aligned}
& \underline{\mathrm{dA}}_{\mathrm{dt}}=\frac{\mathrm{h}}{2} \\
& \mathrm{~A}=\frac{\mathrm{h}}{2} \mathrm{t}
\end{aligned}
$$

where $\quad A=a b$ and $t=T$

$$
\begin{aligned}
& \mathrm{ab}=\frac{\mathrm{h}}{2}(\mathrm{~T}) \\
& \mathrm{T}=\underline{\mathrm{2}} \underline{\mathrm{ab}} \underset{\mathrm{h}}{ }
\end{aligned}
$$

Substituting the values of $a$ and $b$,

$$
T=\frac{2}{2}\left(\frac{p h}{1 e} 2\right)^{3}
$$

From the equation of semi major axis, we have $h=\quad a\left(1 e^{2}\right)$

$$
\mathrm{T}=\mathrm{pa}
$$




\subsection{Eccentricity:}

From equations (34) and (35) we have,

$$
\begin{array}{r}
\frac{\mathrm{re}}{\mathrm{ra}}=\frac{1 \mathrm{e}}{1+\mathrm{e}} \\
\mathrm{e}=\frac{\mathrm{rese}}{\mathrm{catha}}
\end{array}
$$

\subsection{NUMERICAL ILLUSTRATIONS:}

1. A satellite orbits the earth with a perigee radius of $7000 \mathrm{~km}$ and apogee radius of $70000 \mathrm{~km}$. Calculate eccentricity and period of orbit.

Solution:

$$
\begin{gathered}
e=\frac{e x}{c a t h e} \\
=\frac{700007000}{70000+7000} \\
e=0.8182
\end{gathered}
$$

Since e 1, it will be an ellipse.

we know that $r=\frac{h^{2}}{1+e \cos }$

$r_{p}=\frac{h^{2}}{1+e} \frac{1}{1+e}$

$$
7000=\frac{h^{2}}{398600} \frac{1}{1+0: 8182}
$$

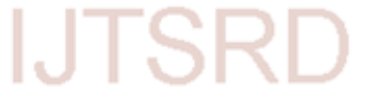

$\mathrm{h}=71226 \mathrm{~km}^{2} / \mathrm{s}$.

Period of orbit, $T={ }^{2} \underline{2}\left(\underline{\mathrm{P}_{1} \mathrm{~h}} \mathrm{e} 2\right)^{3}$

$$
\begin{aligned}
T & \left.=\frac{2}{398600^{2} \quad 10: 8182^{2}}\right)^{3} \\
T= & 7511 \text { seconds } \\
T & =20: 89 \text { hours }
\end{aligned}
$$

2. At two points on a geocentric orbit the altitude and anomaly are $Z_{1}=1700 \mathrm{~km}, 1=$ $130^{\circ}$ and $Z_{2}=500 \mathrm{~km}, 2=50^{\circ}$. Find
a) eccentricity
b) angular momentum
c) perigee radius 
International Journal of Trend in Scientific Research and Development (IJTSRD) ISSN: 2456-6470

d) perigee altitude

e) semi major axis

Solution:

$r_{1}=R_{E}+z_{1}=6378+1700=8078 !(1)$

$r_{2}=R_{E}+z_{2}=6378+500=6878 !(2)$

we know that $r=\frac{h^{2}}{1+e \cos }$

(1) $) 8078=\frac{h^{2}}{(398600)} \frac{1}{1+e \cos 130^{\circ}}$

$h^{2}=3: 219 \times 10^{9} 2: 069 \times 10^{9}$ e $!(3)$

(2) $) 6878=\overline{(398600)} \overline{1+e \cos 50^{\circ}}$

$h^{2}=2: 742 \times 10^{9}+1: 762 \times 10^{9} e !(4)$

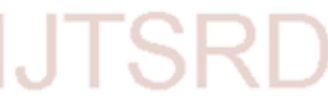

by (3) and (4), $3: 219 \times 10^{9} 2: 069 \times 10^{9} \mathrm{e}=2: 742 \times 10^{9}+1: 762 \times 10^{9} \mathrm{e}$

$3: 219 \quad 2: 742=\mathrm{e}(1: 762 \times 2: 069)$

$e=0: 477=3: 831$

$e=0: 1245$

Since e 1 , it will be an ellipse.

$h^{2}=3: 219 \times 10^{9} 2: 069 \times 10^{9}(0: 1245)$

$h^{2}=2961409500$

$\mathrm{h}=54418 \mathrm{~km}^{2} / \mathrm{s}$

c) $r_{p}=\frac{h_{2}}{1+e} 1$

$r_{p}=\frac{2}{39418^{2}} \frac{1}{1+0: 1245}$


$r_{p}=6607 \mathrm{~km}$

d) $z_{p}=r_{p} \quad R_{E}$

$z_{p}=66076378$

$z_{p}=229 \mathrm{~km}$

e) $r_{a}=\frac{h_{2}}{1} \frac{1}{e}$

$r_{a}=\frac{54418^{2}}{39860010: 1245}$

$r_{a}=8486$

$a=r_{a}+r_{p} 2$

$a=\underline{8486+6607} 2$

$a=7546: 5$

3. At a given point of spacecraft's geocentric trajectory, the perigee alti-tude is $300 \mathrm{~km}$, the speed is $15 \mathrm{~km} / \mathrm{s}$ and the ight path angle is $50^{\circ}$. Show that path is hyperbola and calculate

a) hyperbolic excess speed

b) angular momentum

c) true anomaly

d) eccentricity

Solution:

a) $r_{p}=R_{E}+z_{p}=6378+300=6678$

$$
\begin{aligned}
& v_{\text {esc }}={ }^{q^{2}} \frac{\frac{}{2(398600)}}{6678} \\
& v_{\text {esc }}=q \frac{q}{v_{\text {esc }}=10: 925} \\
& v_{\text {inf } 2}=v^{2}-v_{\text {es }}{ }^{2} \mathrm{c} \\
& =225 \quad 119: 377
\end{aligned}
$$


International Journal of Trend in Scientific Research and Development (IJTSRD) ISSN: 2456-6470

$\mathrm{V}$ inf $^{2}=105: 623$

$v_{\text {inf }}=10: 277 \mathrm{~km}^{2}=\mathrm{s}^{2}$

b) $v_{r}=r \sin =15 \sin 50^{\circ}=11: 49$

$v_{p}=r \cos =15 \cos 50^{\circ}=9: 641$

$\mathrm{h}=\mathrm{rv} \mathrm{p}=6678(9: 461)$

$\mathrm{h}=64383 \mathrm{~km}^{2} / \mathrm{s}$

c) we know that $r=$

$6678=\underline{64383^{2}} 1$

$3986001+e \cos$

e $\cos =0: 5573 !(1)$

$v=e \sin$

$r \quad h$

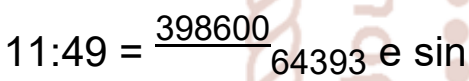

e $\sin =1: 856$ ! (2)

$\tan =\underline{e} \underline{\sin }$

$\tan =0^{1:}: 5573^{856}$

$=73: 28^{\circ}$

d) (1) )e $\cos 73: 28^{\circ}=0: 5573$

$e=1: 9379$

Since e 1, it will be hyperbolic orbit.

(5). The perigee of a satellite in a parabolic trajectory is $9000 \mathrm{~km}$. Find the distance $\mathrm{d}$ between points $P_{1}$ and $P_{2}$ on orbit which are $11000 \mathrm{~km}$ and $22000 \mathrm{~km}$ from the center of earth.

Solution: We know that, $\mathrm{r}_{\mathrm{p}}=\underline{\mathrm{h}_{2}} \underline{\underline{1}}$ 
2.8/sum5.png

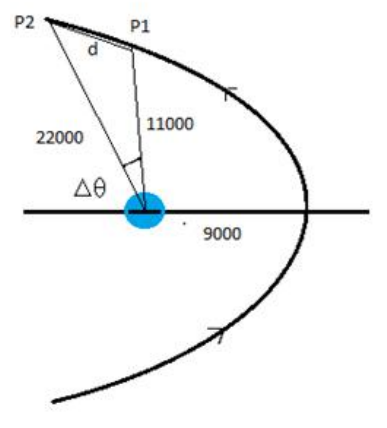

Figure 1: parabola

Since it is a parabolic trajectory its eccentricity will be one.

$$
\begin{aligned}
& r_{p}=\frac{h^{2}}{1+1} \frac{1}{r_{p}}={ }_{2} h_{2} \\
& h=p_{2} \overline{r_{p}} \\
& h=\frac{g}{2(398600)(9000)} \\
& h=84704 \mathrm{~km}^{2} / \mathrm{s}
\end{aligned}
$$

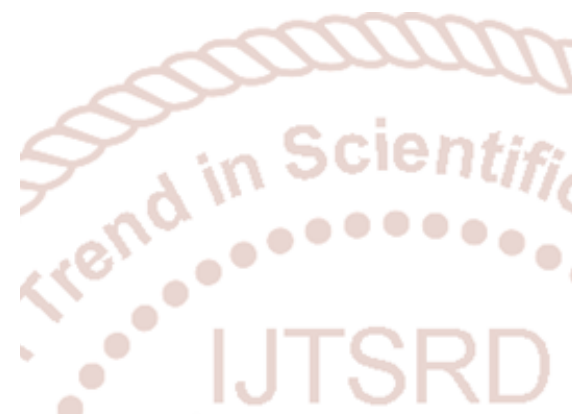

True anomalies of points $P_{1}$ and $P_{2}$ are found using orbit equation,

$$
\text { we know that } r=\frac{h^{2}}{1+\frac{1}{e c o s}}
$$

$$
11000=\frac{84704^{2}}{398600} \frac{1}{1+\cos 1}
$$

$1=50: 48^{\circ}$

$$
\begin{aligned}
& 22000=\frac{84704^{2}}{398600} \frac{1}{1+\cos 2} \\
& 2=100: 48^{\circ} \\
& =2 \quad 1=100: 48^{\circ} \quad 50: 48^{\circ} \\
& =50^{\circ}
\end{aligned}
$$

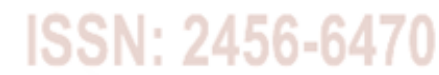

From law of cosines from trignometry,

$d^{2}=11000^{2}+22000^{2} 2(11000)(22000) \cos$

$50^{\circ} \mathrm{d}=17143 \mathrm{~km}$ 


\section{REFERENCE}

1. Orbital Mechanics 2nd Edition by John E. Prussing, Bruce A. Con-way.

2. Modern Astrodynamics Second Edition 2nd Edition by William E.

3. Fundamentals of Astrodynamics by Bate and Mueller.

4. Classical Mechanics by N C Rana and P S Joag.

5. Mechanics by L D Landau and E M Lifhitz.

6. Mathematical Methods of Classical Mechanics by V I Arnold.

7. Practical Conic Sections by J. W. Downs.

8. Coordinate Geometry By SK Goyal.

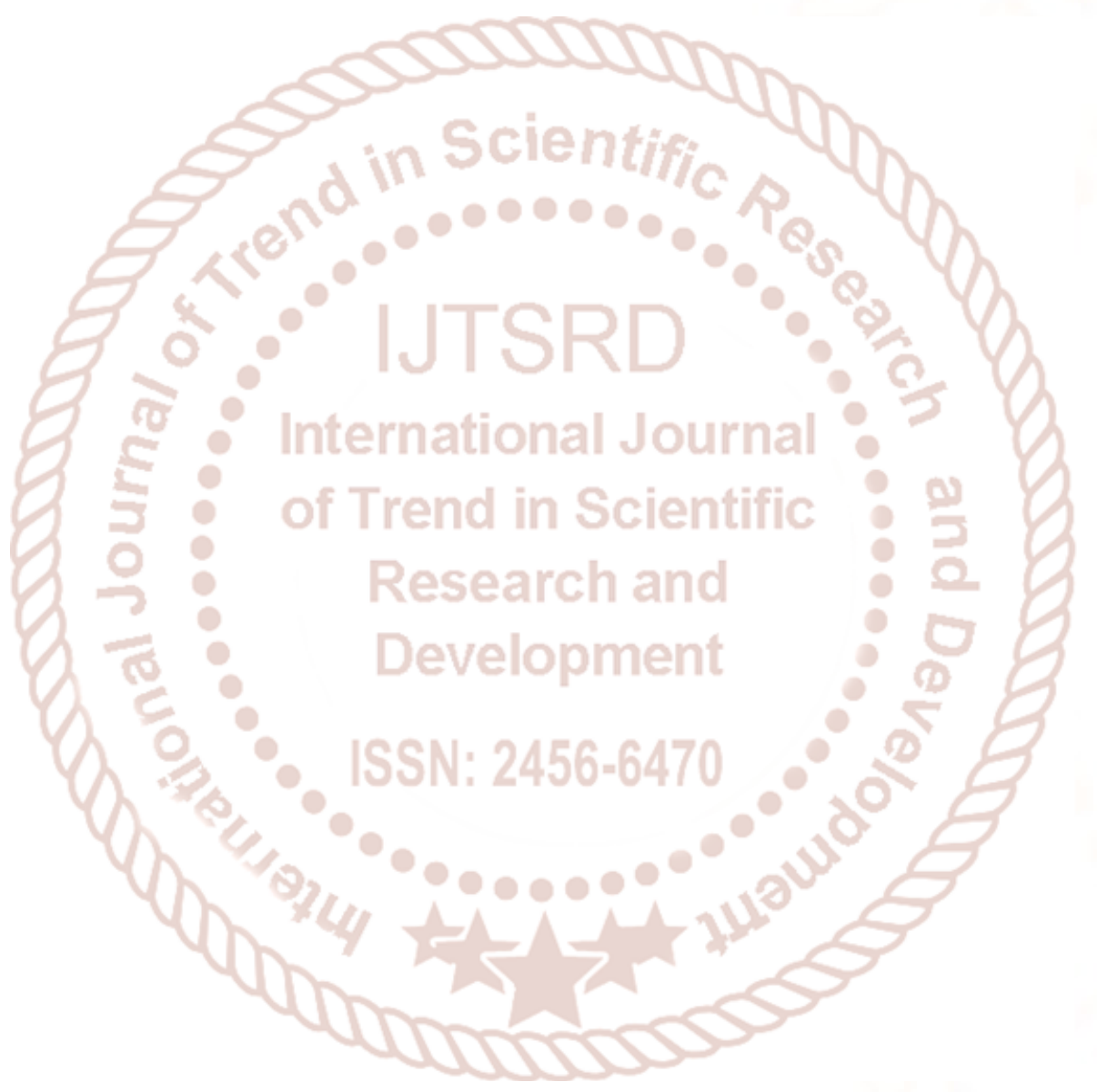

